JOURNAL OF THE AMERICAN MATHEMATICAL SOCIETY

Volume 20, Number 1, January 2007, Pages 211-217

S $0894-0347(06) 00539-X$

Article electronically published on June 19, 2006

\title{
REPRESENTATIONS OF AFFINE HECKE ALGEBRAS AND BASED RINGS OF AFFINE WEYL GROUPS
}

\author{
NANHUA XI
}

It is known that an interesting part of the study of the representation theory of $p$-adic groups can be reduced to the study of the representation theory of affine Hecke algebras [B, $\mathrm{V}]$. Let $(W, S)$ be an extended affine Weyl group and $H_{k, q_{0}}$ the corresponding Hecke algebra over a field $k$ with a nonzero parameter $q_{0} \in k$. When $k$ is the complex numbers field and $q_{0}$ is not a root of unity, a classification of simple representations of $H_{k, q_{0}}$ was established in KL2] (Deligne-LanglandsLusztig classification). For affine type $A$, a classification of simple representations of $H_{k, q_{0}}$ was obtained in [AM] for any $q_{0}$ and arbitrary sufficiently large $k$. When $k$ is algebraically closed and has positive characteristic, the representations of $H_{k, q_{0}}$ were studied by Vignéras, as part of her study of modular representations of $p$-adic groups [V]. In this paper we shall verify a conjecture of Lusztig [L6, 7(a)] by means of the based ring of an extended affine Weyl group (Theorem 3.3). The conjecture says that if the parameter $q_{0}$ is not a root of the corresponding Poincaré polynomial, then the classification established in KL2] remains valid. The restriction is necessary for the classification; see Remark 3.4 (a).

\section{Extended AFFine Weyl groups And their HeCke Algebras}

1.1. Let $G$ be a connected reductive group over the field $\mathbf{C}$ of complex numbers with simply connected derived group and $T$ a maximal torus of $G$. Let $N_{G}(T)$ be the normalizer of $T$ in $G$. Then $W_{0}=N_{G}(T) / T$ is a Weyl group, which acts on the character group $X=\operatorname{Hom}\left(T, \mathbf{C}^{*}\right)$ of $T$. The semi-direct product $W=W_{0} \ltimes X$ is called an extended affine Weyl group. We shall denote by $S$ the set of simple reflections of $W$.

Denote by $H_{k, q_{0}}$ the Hecke algebra of $(W, S)$ over an arbitrary field $k$ with a nonzero parameter $q_{0} \in k$. We shall assume that $k$ contains the square roots of $q_{0}$. The following result is due to J. Bernstein; see [L1, Theorem 8.1] for a proof.

(a) The center $Z$ of $H_{k, q_{0}}$ is a finitely generated $k$-algebra and $H_{k, q_{0}}$ is a finitely generated $Z$-module.

The following result was proved in [KL2, Proof of Prop. 5.13] when $k$ is uncountable, by using an argument of Dixmier.

Proposition 1.2. Any simple $H_{k, q_{0}}$-module is finite dimensional.

Proof. Let $M$ be a simple $H_{k, q_{0}}$-module and $\mathcal{D}=\operatorname{End}_{H_{k, q_{0}}} M$. Then $\mathcal{D}$ is a division ring. For $z$ in $Z$, let $f_{z}: M \rightarrow M, m \rightarrow z m$. Then $f_{z}$ is in $\mathcal{D}$ and the map

Received by the editors February 10, 2005.

2000 Mathematics Subject Classification. Primary $20 \mathrm{C} 08$.

Key words and phrases. Affine Hecke algebra, based ring, representation.

The author was partially supported by a fund of the 973 Program.

(C)2006 American Mathematical Society Reverts to public domain 28 years from publication 
$f: Z \rightarrow \mathcal{D}, z \rightarrow f_{z}$ is a homomorphism of $k$-algebras. Let $Y=f(Z)$. By section 1.1 (a), $Y$ is a finitely generated $k$-algebra. We only need to show that each element in $Y$ is algebraic over $k$.

Let $r$ be the transcendency degree of $Y$ over $k$. By the Noether normalization theorem, there are elements $y_{1}, \ldots, y_{r}$ in $Y$ such that $Y$ is integral over $k\left[y_{1}, \ldots, y_{r}\right]$.

We need to show that $r$ is zero. Assume that $r \geq 1$. Note that $y_{1}^{-1}$ is not in $Y$ since $y_{1}, \ldots, y_{r}$ are algebraically independent and $Y$ is integral over $k\left[y_{1}, \ldots, y_{r}\right]$. By section $1.1(\mathrm{a}), M$ is a finitely generated $Z$-module. Let $v_{1}, \ldots, v_{g}$ be elements in $M$ which generate $M$ as a $Z$-module. Choose $x$ in $Z$ such that $f_{x}=y_{1}$. Since $y_{1}$ is invertible in $\mathcal{D}$, we can find $u_{i}$ in $M$ such that $v_{i}=x u_{i}$ for all $i$. Let $u_{i}=\sum_{j} \xi_{j i} v_{j}, \xi_{j i} \in Z$. Set $\eta_{j i}=x \xi_{j i}$ if $j \neq i$, and $\eta_{i i}=1-x \xi_{i i}$. Then we have $\operatorname{det}\left(\eta_{i j}\right) v_{i}=0$ for all $i$. But $\operatorname{det}\left(\eta_{i j}\right)=1-x z$ for some $z$ in $Z$. Thus $f_{1-x z}=1-f_{x} f_{z}=1-y_{1} f_{z}=0$. This implies that $y_{1}$ is invertible in $Y$ and leads to a contradiction. Therefore we must have $r=0$. The proposition is proved.

\section{2. $a$-FUNCTION AND BASED RING}

In this section we will see that the simple $J_{k}$-modules and simple $H_{k, q_{0}}$-modules have a nice relationship.

2.1. We refer to [L2, 2.1] and [L3, 2.3] for the definitions of the function $a: W \rightarrow \mathbf{N}$ and of the based ring $J$ of $W$ respectively. Following [L3] we denote by $t_{w}, w \in W$ the basis elements of $J$. For each nonnegative integer $i$ we denote by $J^{i}$ the subgroup of $J$ generated by all $t_{w}$ with $a(w)=i$. Then $J^{i}$ is a two-sided ideal of $J$ and $J$ is the direct sum of all $J^{i}$. Set $J_{k}=J \otimes_{\mathbf{z}} k$ and $J_{k}^{i}=J^{i} \otimes_{\mathbf{z}} k$. Thus $J_{k}^{i}$ is a direct summand of $J_{k}$ and is also a $k$-algebra. By abusing notation we also write $t_{w}$ for $t_{w} \otimes 1$.

Let $C_{w}, w \in W$ be the Kazhdan-Lusztig basis of $H_{k, q_{0}}$ in [KL1, L4] and write $C_{w} C_{u}=\sum h_{w, u, v} C_{v}, h_{w, u, v} \in k$. Let $D$ be the set of distinguished involutions of $W$. The following properties are due to Lusztig; see [L3, 2.4 (a)] and [L4, Prop. 1.7, Prop. 1.6 (i), (ii)].

(a) There is a well-defined homomorphism $\varphi: H_{k, q_{0}} \rightarrow J_{k}$ of $k$-algebras such that

$$
\varphi\left(C_{w}\right)=\sum_{\substack{d \in D \\ u \in W \\ a(d)=a(u)}} h_{w, d, u} t_{u}, \quad w \in W .
$$

(b) The homomorphism $\varphi$ in (a) is injective. Thus $H_{k, q_{0}}$ can be regarded as a subalgebra of $J_{k}$ by means of $\varphi$.

(c) The center $Z\left(J_{k}\right)$ of $J_{k}$ is a finitely generated $k$-algebra and $J_{k}$ is a finitely generated $Z\left(J_{k}\right)$-module.

(d) There is a well-defined right $H_{k, q_{0}}$-module structure on $J_{k}^{i}$ such that

$$
t_{w} C_{u}=\sum_{\substack{v \in W \\ a(v)=a(w)}} h_{w, u, v} t_{v} .
$$

In this way, $J_{k}^{i}$ becomes a $J_{k}-H_{k, q_{0}}$-bimodule. See [L4, 1.4 (b)].

The following result was proved by Lusztig [L4, Prop. 1.6 (iii)] provided that $k$ is uncountable.

Lemma 2.2. Any simple $J_{k}$-module is finite dimensional. 
Proof. A proof is similar to that for Proposition 1.2.

2.3. Let $E$ be a $J_{k}$-module through the homomorphism $\varphi$, it is endowed with an $H_{k, q_{0}}$-module structure. We denote the $H_{k, q_{0}}$-module by $E_{\varphi}$. Convention: For any subset $N$ of $E$ and any subset $L$ of $H_{k, q_{0}}$, we often write $L N$ for $\varphi(L) N$. Thus, as a set the notation $L N$ is unambiguous, no matter whether $N$ is regarded as a subset of $E$ or as a subset of $E_{\varphi}$.

For each simple $J_{k}$-module $E$, there is a unique $i$ such that $J_{k}^{i} E=E$. We define $a(E)$ to be $i$. For an integer $i$, we denote by $H_{k, q_{0}}^{\geq i}\left(\right.$ resp. $\left.H_{k, q_{0}}^{>i}\right)$ the subspace of $H_{k, q_{0}}$ spanned by all $C_{w}$ with $a(w) \geq i$ (resp. $a(w)>i$ ). Both $H_{k, q_{0}}^{\geq i}$ and $H_{k, q_{0}}^{>i}$ are two-sided ideals of $H_{k, q_{0}}$. For each $H_{k, q_{0}}$-module $M$ we then define $a(M)$ to be $i$ if $H_{k, q_{0}}^{\geq i} M \neq 0$ but $H_{k, q_{0}}^{>i} M=0$.

Let $M$ be an $H_{k, q_{0}}$-module with $a(M)=i$. We define $\tilde{M}$ to be $J_{k}^{i} \otimes_{H_{k, q_{0}}} M$; here we regard $J_{k}^{i}$ as a $J_{k}-H_{k, q_{0}}$-bimodule as in section 2.1 (d). Then $\tilde{M}$ is a $J_{k}$-module. There is a natural homomorphism of $H_{k, q_{0}}$-modules $p: \tilde{M}_{\varphi} \rightarrow M, t_{w} \otimes m \rightarrow C_{w} m$. We have ([L4, Proof of Lemma 1.9]).

(a) When $M$ is simple, the map $p$ is surjective and $C_{w} \operatorname{ker} p=0$ whenever $a(w) \geq$ $a(M)$.

The following assertion is clear.

(b) Let $E$ be a simple $J_{k}$-module. Then $H_{k, q_{0}}^{>a(E)} E_{\varphi}=0$. In particular, $a(M) \leq$ $a(E)$ for any simple constituent $M$ of $E_{\varphi}$. Also for any subset $N$ of $E$ or $E_{\varphi}$, $H_{k, q_{0}}^{\geq a(E)} N$ is spanned by all $C_{w} N, w \in W$ with $a(w)=a(E)$.

Lemma 2.4. Let $E$ be a simple $J_{k}$-module and $N$ a submodule of $E_{\varphi}$ such that $C_{w} N \neq 0$ for some $w \in W$ with $a(w)=a(E)$. Regarding $N$ as a subset of $E$, then $H_{k, q_{0}}^{\geq a(E)} N=E$. In particular, $N=E_{\varphi}$ as $H_{k, q_{0}}$-modules.

Proof. Using section 2.3 (b) we know $a(N)=a(E)$. Thus $\tilde{N}=J_{k}^{a(E)} \otimes_{H_{k, q_{0}}} N$. We have a well-defined $k$-linear map

$$
\theta: \tilde{N} \rightarrow E, t_{w} \otimes v \rightarrow \varphi\left(C_{w}\right) v .
$$

Using [L3, 2.4 (c)] we see that $\theta$ is a homomorphism of $J_{k}$-modules. Since $E$ is a simple $J_{k}$-module and $\theta(\tilde{N})=H_{k, q_{0}}^{\geq a(E)} N \neq 0$, we must have $H_{k, q_{0}}^{\geq a(E)} N=E$. The lemma is proved.

Lemma 2.5. Let $E$ be a simple $J_{k}$-module. Then

(a) $E_{\varphi}$ has at most one simple constituent $M$ such that $a(M)=a(E)$.

(b) If $E_{\varphi}$ has a simple constituent $M$ such that $a(M)=a(E)$, then $M$ is a quotient module of $E_{\varphi}$.

(c) If $E_{\varphi}$ has a simple constituent $M$ such that $a(M)=a(E)$, then $M$ is the unique simple quotient module of $E_{\varphi}$.

Proof. Assume that $E_{\varphi}$ has a simple constituent $M$ such that $a(M)=a(E)$. Let $N_{2} \subset N_{1}$ be two submodules of $E_{\varphi}$ such that the quotient module $N_{1} / N_{2}$ is $M$. Then $C_{w} N_{1} \neq 0$ for some $w \in W$ with $a(w)=a(E)$. By Lemma 2.4 we have $N_{1}=E_{\varphi}$. Since $H_{k, q_{0}}^{\geq a(E)}$ is a two-sided ideal, using Lemma 2.4 we see that $N_{2}=$ $\left\{v \in E_{\varphi} \mid H_{k, q_{0}}^{\geq a(E)} v=0\right\}$.

(a) and (b) follow. 
Now we argue for (c). Let $N$ be a maximal submodule of $E_{\varphi}$. Using Lemma 2.4 we see that $N$ is a submodule of $N_{2}=\left\{v \in E_{\varphi} \mid H_{k, q_{0}}^{\geq a(E)} v=0\right\}$. By the argument for (a) and (b), $N_{2}$ is a maximal submodule of $E_{\varphi}$. Thus $N=N_{2}$ and $E_{\varphi} / N=M$ is the unique simple quotient module of $E_{\varphi}$.

The lemma is proved.

Corollary 2.6. Let $E$ be a simple $J_{k}$-module. Then $E_{\varphi}$ has a simple constituent $M$ with $a(M)=a(E)$ if and only if $C_{w} E_{\varphi} \neq 0$ for some $w$ with $a(w)=a(E)$. In this case $E_{\varphi}$ has a unique maximal submodule.

Proof. The "only if" part is obvious. Now we prove the "if" part. Assume that $E_{\varphi}$ had no simple constituent $M$ with $a(M)=a(E)$. Let $N$ be a maximal submodule of $E_{\varphi}$. Then $E_{\varphi} / N$ is simple. By assumption and section 2.3 (b), we have $H_{k, q_{0}}^{\geq a(E)} E_{\varphi} \subset N$. However, $C_{w} E_{\varphi} \neq 0$ for some $w$ with $a(w)=a(E)$. By Lemma 2.4 we have $H_{k, q_{0}}^{\geq a(E)} E_{\varphi}=E_{\varphi}$. This is a contradiction. The corollary is proved.

Lemma 2.7. Let $E$ and $E^{\prime}$ be two simple $J_{k}^{i}$-modules. Assume that $E_{\varphi}$ (resp. $E_{\varphi}^{\prime}$ ) has a simple quotient $M$ (resp. $\left.M^{\prime}\right)$ such that $a(M)=i\left(\right.$ resp. $\left.a\left(M^{\prime}\right)=i\right)$. Then $M$ is isomorphic to $M^{\prime}$ if and only if $E$ is isomorphic to $E^{\prime}$.

Proof. Let $\pi: E_{\varphi} \rightarrow M$ be the natural projection. Since $H_{k, q_{0}}^{\geq i} E_{\varphi} \neq 0$, by section 2.3 (b) we have $\widetilde{E_{\varphi}}=J_{k}^{i} \otimes_{H_{k, q_{0}}} E_{\varphi}$. For simplicity, we shall write $\tilde{E}$ for $\widetilde{E_{\varphi}}$. There are two well-defined $k$-linear maps

$$
\begin{gathered}
p^{\prime}: \tilde{E} \rightarrow \tilde{M}, t_{w} \otimes v \rightarrow t_{w} \otimes \pi(v), \\
\theta: \tilde{E} \rightarrow E, t_{w} \otimes v \rightarrow \varphi\left(C_{w}\right) v .
\end{gathered}
$$

Clearly $p^{\prime}$ is a homomorphism of $J_{k}$-modules. According to the proof of Lemma 2.4, $\theta$ is also a homomorphism of $J_{k}$-modules. Obviously we have $\pi \theta=p p^{\prime}$ (see section 2.3 for the definition of $\left.p: \tilde{M}_{\varphi} \rightarrow M\right)$.

Since $p^{\prime}$ is a surjection, the homomorphism $p^{\prime}$ induces a surjective homomorphism of $J_{k}$-modules, $\bar{p}^{\prime}: \tilde{E} / \operatorname{ker} \theta \rightarrow \tilde{M} / p^{\prime}(\operatorname{ker} \theta)$. As $J_{k^{-}}$modules, $\tilde{E} / \operatorname{ker} \theta$ is isomorphic to $E$, since $E$ is simple and $\theta(\tilde{E})=H_{\vec{k}, q_{0}}^{\geq i} E \neq 0$. Thanks to $\pi \theta=p p^{\prime}$, we know that $p^{\prime}(\operatorname{ker} \theta)$ is in the kernel of $p$. By section 2.3 (a), $\operatorname{ker} p \varsubsetneqq \tilde{M}$, so $\bar{p}^{\prime}$ is an isomorphism and $E$ is isomorphic to $\tilde{M} / p^{\prime}(\operatorname{ker} \theta)$.

By section 2.3 (a), $H_{k, q_{0}}^{\geq i} \operatorname{ker} p=0$; hence we have $H_{k, q_{0}}^{\geq i} p^{\prime}(\operatorname{ker} \theta)=0$. Thus $E$ can be characterized as the unique simple constituent $F$ of the $J_{k}$-module $\tilde{M}$ such that $H_{k, q_{0}}^{\geq i} F_{\varphi} \neq 0$.

As a consequence, if $M$ is isomorphic to $M^{\prime}$, then $E$ must be isomorphic to $E^{\prime}$. The lemma is proved.

Corollary 2.8 ([L4, Corollary 3.6]). Assume that for each simple $J_{k}^{i}$-module E, the $H_{k, q_{0}}$-module $E_{\varphi}$ has a simple constituent $M$ with $a(M)=i$. Then both of the $J_{k}$-modules $\tilde{E}$ and $\tilde{M}$ are isomorphic to $E$.

Proof. By Lemma 2.5 (c), $M$ is the unique simple quotient of $E_{\varphi}$. Note that $J_{k}^{r} \tilde{E}=0$ if $r \neq i$ (recall that $\tilde{E}$ stands for $\widetilde{E_{\varphi}}$ ). Let $\theta: \tilde{E} \rightarrow E$ be as in the proof of Lemma 2.7. As in the proof of [L4, Lemma 1.9], one may check that $C_{w} \operatorname{ker} \theta=0$ whenever $a(w) \geq i$. If $\operatorname{ker} \theta \neq 0$, then by assumption, $C_{w} \operatorname{ker} \theta \neq 0$ for some $w$ with $a(w)=i$. This yields a contradiction. Therefore $\operatorname{ker} \theta=0$ and as $J_{k}$-modules, 
$\tilde{E}$ is isomorphic to $E$. By the proof of Lemma 2.7 we know that $\tilde{E}$ and $\tilde{M}$ are isomorphic in this case. The corollary is proved.

\section{MAin RESUlts}

In this section we give our main results.

Denote by $W^{I}$ the subgroup of $W$ generated by a subset $I$ of $S$ and call it a parabolic subgroup. Let $J_{k}^{I}$ be the subspace spanned by all $t_{w}, w \in W^{I}$.

Theorem 3.1. Assume that char $k=0$. Then as a two-sided ideal, $J_{k}$ is generated by all $J_{k}^{I}$ for all finite parabolic subgroups $W^{I}$.

Proof. According to [L5, Theorem 4.2] and [L5, Theorem 6.7(a2)], for any simple $J_{\mathbf{C}}$-module $E$, we can find a finite parabolic subgroup $W^{I}$ of $W$ such that the action of $J_{\mathbf{C}}^{I}$ on $E$ is nonzero. This implies that as a two-sided ideal, $J_{\mathbf{C}}$ is generated by all $J_{\mathbf{C}}^{I}$ for all finite parabolic subgroups $W^{I}$. With respect to the basis $\left\{t_{w} \mid w \in W\right\}$, the structure constants of $J_{k}$ are in $\mathbf{N}$ if char $k=0$. The theorem follows.

When $q_{0}$ is not a root of unity, the following result was proved by Lusztig [L4, Theorem 3.4], except for the uniqueness in (a).

Theorem 3.2. Assume that $\operatorname{char} k=0$ and $\sum_{w \in W_{0}} q_{0}^{l(w)} \neq 0$ (l is the length function of $W$ ). Then

(a) for each simple $J_{k}$-module $E$, the $H_{k, q_{0}}$-module $E_{\varphi}$ has a unique simple constituent $M$ such that $a(M)=a(E)$. For other simple constituents $M^{\prime}$ of $E_{\varphi}$ we have $a\left(M^{\prime}\right)<a(E)$. The $H_{k, q_{0}}$-module $M$ is the unique simple quotient of $E_{\varphi}$. (The uniqueness is part of [L2, 9.10, Conjecture A]. The other part of the conjecture was proved in [L3.)

(b) Keep the notation in (a). The map $E \rightarrow M$ defines a bijection between the isomorphism classes of simple $J_{k}$-modules and the isomorphism classes of simple $H_{k, q_{0}}$-modules.

Proof. Let $W^{I}$ be a finite parabolic subgroup of $W$. Since $\sum_{w \in W_{0}} q_{0}^{l(w)} \neq 0$, it is easy to check that $\sum_{w \in W^{I}} q_{0}^{l(w)} \neq 0$. Thus the subalgebra $H_{k, q_{0}}^{I}$ of $H_{k, q_{0}}$ generated by all $C_{w}\left(w \in W^{I}\right)$ is semisimple [G1, Theorem 3.9]. Then the restriction of $\varphi$ to $H_{k, q_{0}}^{I}$ induces an isomorphism $\varphi_{I}: H_{k, q_{0}}^{I} \rightarrow J_{k}^{I}$ [G2, Lemma 2.1]. The isomorphism $\varphi_{I}$ sends $C_{w}\left(w \in W^{I}\right)$ to a linear combination of $t_{u}, u \in W^{I}$ with $a(u) \geq a(w)$.

Now for each simple $J_{k}$-module $E$, we can find a finite parabolic subgroup $W^{I}$ such that $J_{k}^{I} E \neq 0$ (Theorem 3.1). Let $N_{1}=J_{k}^{I} E$ and $N_{2}=\left\{v \in E \mid J_{k}^{I} v=0\right\}$. Then $E=N_{1} \oplus N_{2}$ and $J_{k}^{I} N_{1}=N_{1}$. Moreover, for any $v$ in $N_{1}$ and $h$ in $H_{k, q_{0}}^{I}$, we have $\varphi(h) v=\varphi_{I}(h) v$. Let $u \in W^{I}$ be such that $t_{u} N_{1} \neq 0$. Then $a(u)=a(E)$ and $h=\varphi_{I}^{-1}\left(t_{u}\right)$ is a linear combination of $C_{w}, w \in W^{I}$ with $a(w) \geq a(E)$. Now we have $h N_{1}=\varphi(h) N_{1}=\varphi_{I}(h) N_{1}=t_{u} N_{1} \neq 0$. Using section 2.3 (b) we can find an element $w \in W^{I}$ such that $a(w)=a(E)$ and $C_{w} N_{1} \neq 0$. This implies that $C_{w} E_{\varphi} \neq 0$. By Corollary 2.6 and Lemma 2.5 , we see that $E_{\varphi}$ has a unique simple constituent $M$ such that $a(M)=a(E)$. Moreover, $M$ is the unique simple quotient of $E_{\varphi}$.

Using section 2.3 (b), we know that for other simple constituents $M^{\prime}$ of $E_{\varphi}$ we have $a\left(M^{\prime}\right)<a(E)$. Part (a) is proved.

Using section 2.3 (a) and Lemma 2.7 we see that (b) is true. 
Theorem 3.3. Assume that $k=\mathbf{C}$ and $\sum_{w \in W_{0}} q_{0}^{l(w)} \neq 0$. Then the classification of simple $H_{k, q_{0}}$-modules in KL2 remains valid.

Proof. The theorem follows from [L5, Theorem 4.2] and Theorem 3.2 (b).

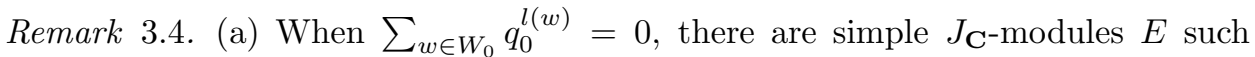
that the $H_{k, q_{0}}$-modules $E_{\varphi}$ have no simple constituents $M$ with $a(M)=a(E)$ [X1, Theorem 7.8].

(b) A weaker result was proved in [X1, Theorem 6.6].

(c) In $\mathrm{Gr}$, Grojnowski announced a stronger result. The proof seems to not be available yet. The validity of the result will be commented on in a future work.

(d) For type $\tilde{A}_{n}$, rank 2 cases, the structure of the based ring $J$ is known explicitly X1, X2, BO. In these cases we can get a classification of simple $H_{k, q_{0}}$-modules for any field $k$ containing square roots of $q_{0}$, by means of $J_{k}$. The result suggests that an analogue of the Deligne-Langlands-Lusztig classification of simple $H_{k, q_{0}}$ modules remains true, provided that $k$ is algebraically closed and the subalgebra $H\left(W_{0}\right)_{k, q_{0}}$ of $H_{k, q_{0}}$ generated by all $C_{w}\left(w \in W_{0}\right)$ is semisimple. The details will appear elsewhere.

\section{ACKNOWLEDGEMENTS}

I am grateful to Professors T. Tanisaki and Y. Zhu for stimulating conversations. Part of the work was done during my visit to the Hong Kong University of Science and Technology and to the Osaka City University. I thank the universities for their hospitality and for financial support. I would like to thank the referee for very helpful comments.

\section{REFERENCES}

[AM] S. Ariki and A. Mathas, The number of simple modules of the Hecke algebras of type $G(r, 1, n)$, Math. Z. 233 (2000), 601-623. MR1750939(2001e:20007)

[BO] R. Bezrukavnikov and V. Ostrik, On tensor categories attached to cells in affine Weyl groups, II, in "Representations of algebraic groups and quantum groups", Advanced Studies in Pure Math., vol. 40, Math. Soc. of Japan, Tokyo, 2004, pp. 101-119. MR2074591 (2006e:20006)

[B] A. Borel, Admissible representations of a semi-simple group over a local field with vectors fixed under an Iwahori subgroup, Invent. Math. 35 (1975), 233-259. MR0444849 (56:3196)

[Gr] I. Grojnowski, Representations of affine Hecke algebras and affine quantum GL $_{n}$ at roots of unity, Inter. Math. Res. Notices 5 (1994), 213-216. MR1270135 (95e:20054)

[G1] A. Gyoja, Modular representation theory over a ring of higher dimension with application to Hecke algebras, J. Alg. 174 (1995), 553-572. MR.1334224 (96m:20024)

[G2] A. Gyoja, Cells and modular representations of Hecke algebras, Osaka J. Math. 33 (1996), no. 2, 307-341. MR 1416051 (97k:20018)

[KL1] D. Kazhdan and G. Lusztig, Representations of Coxeter groups and Hecke algebras, Invent. Math. 53 (1979), 165-184. MR0560412 (81j:20066)

[KL2] D. Kazhdan and G. Lusztig, Proof of the Deligne-Langlands conjecture for Hecke algebras, Invent. Math. 87 (1987), no. 1, 153-215. MR0862716 (88d:11121)

[L1] G. Lusztig, Singularities, character formulas, and a q-analog of weight multiplicities, Astérisque 101-102 (1983), pp. 208-227. MR0737932 (85m:17005)

[L2] G. Lusztig, Cells in affine Weyl groups, in "Algebraic groups and related topics", Advanced Studies in Pure Math., vol. 6, Kinokunia and North Holland, 1985, pp. 255-287. MR0803338 (87h:20074)

[L3] G. Lusztig, Cells in affine Weyl groups, II, J. Alg. 109 (1987), 536-548. MR0902967 $(88 \mathrm{~m}: 20103 \mathrm{a})$ 
[L4] G. Lusztig, Cells in affine Weyl groups, III, J. Fac. Sci. Univ. Tokyo Sect. IA Math. 34 (1987), 223-243. MR0914020 (88m:20103b)

[L5] G. Lusztig, Cells in affine Weyl groups, IV, J. Fac. Sci. Univ. Tokyo Sect. IA Math. 36 (1989) No. 2, 297-328. MR.1015001 (90k:20068)

[L6] G. Lusztig, Representations of affine Hecke algebras, Astérisque 171-172 (1989), 73-84. MR $1021500(90 \mathrm{k}: 22028)$

[V] M.-V. Vignéras, Modular representations of p-adic groups and of affine Hecke algebras, Proc. of Inter. Congress. Math., Beijing 2002, Vol. 2, pp. 667-677, Higher Eduction Press, 2002. MR 1957074 (2004i:22019)

[X1] N. Xi, Representations of affine Hecke algebras, LNM 1587, Springer-Verlag, Berlin, 1994. MR.1320509 (96i:20058)

[X2] N. Xi, The based ring of two-sided cells of affine Weyl groups of type $\tilde{A}_{n-1}$, Mem. of Amer. Math. Soc., Vol. 157, No. 749, 2002. MR.1895287(2003a:20072)

Institute of Mathematics, Chinese Academy of Sciences, Beijing 100080, People's Republic of China

E-mail address: nanhua@math.ac.cn 